\title{
A High-Sensitivity Particle Monitor Using an Integration Sphere
}

\author{
Kenzo Watanabe, Fellow, IEEE, Tomuo Yamaguchi, Masakatsu Nakayama, Yi-Zhu Gao, Tadashi Nagasawa, and \\ Taiyo Ozawa
}

\begin{abstract}
A particle monitor is developed for real-time measurement of dust concentration measured in living and working environments. The principle of the device is based on the optical scattering method. An integration sphere is used to collect the light scattered by dust onto a photo-detector (PD). A double-modulated laser system, a low-noise current-to-voltage converter and a synchronous demodulator for averaging are used for the optical source and detector, respectively, to enhance the signal-to-noise ratio (SNR). Individual performances of the optical and signal processing units indicate that a dust concentration lower than 0.2 $\mu \mathrm{g} / \mathrm{m}^{3}$ is detectable, but the minimum detectable concentration of an assembled prototype monitor is limited to $5 \mu \mathrm{g} / \mathrm{m}^{3}$. This may be due to diffracted light and optical misalignment.
\end{abstract}

Index Terms-Air environment assessment, dust concentration, integration sphere, laser diode, optical scattering, photo-detection, SNR improvement.

\section{INTRODUCTION}

$\mathbf{H}$ UMAN activity is increasingly destroying environmental conditions of the earth. Specifically, contamination of air and water due to toxic gases such as $\mathrm{NO}_{x}, \mathrm{SO}_{x}$, and $\mathrm{CO}_{2}$ presents one of the biggest problems, which should be solved urgently. In addition to toxic gases, the dust produced by over-cultivation and forest fires also influences the climate through the competing effect of cooling the earth due to scattering of visible light and warming the earth due to infrared absorption [1]. Under these circumstances, continuous assessment of the conditions of living and working environments is needed for human comfort and safety [2], [3]. The regulated environments include temperature, relative humidity, air flow for thermal condition, carbon monoxide and carbon dioxide levels, and dust concentrations for the air condition assessments. The work described in this paper is concerned with the dust measurements.

Dust specified in the air condition assessments refers to fine particles smaller than $1 \mu \mathrm{m}$ in diameter in suspension in the atmosphere. The average dust concentration in an ordinary living atmosphere ranges from 40 to $80 \mu \mathrm{g} / \mathrm{m}^{3}$. Typical home dust includes fibers, pollens, ticks, and molds that cause allergic symptoms. In a house close to a heavy traffic road, the dust concentration can far exceed the average. In heavy concentrations, dust including exhaust gases is harmful to human health, causing

Manuscript received May 26, 1999; revised April 22, 2000.

K. Watanabe, T. Yamaguchi, M. Nakayama, and Y.-Z. Gao are with the Research Institute of Electronics, Shizuoka University, Hamamatsu 432-8011, Japan.

T. Nagasawa and T. Ozawa are with the Shizuoka Industrial Research Institute of Shizuoka Prefecture, Shizuoka 421-1298, Japan.

Publisher Item Identifier S 0018-9456(00)05957-X. chest troubles and asthma. The flammable dust in industries and mines is much more dangerous. It causes explosions under the high pressure. Taking these harmful effects into consideration, the National Ambient Air Quality (NAAQ) Standards in the United States and the Health Management Standards in Japan set $150 \mu \mathrm{g} / \mathrm{m}^{3}$ as the maximum allowable dust concentration in working environments and mandate its continuous monitoring.

A standard way of measuring the dust concentration in the atmosphere is to weigh or count the dust piled up onto a filter [4], [5]. This method allows for high-accuracy in measurement, but its application for the real-time measurements is difficult. A conventional method for the real-time measurements is to detect the light scattered by the dust.

The particle size of dust in air is comparable to the laser wavelength, and the scattering due to dust belongs to the Mie scattering. The Mie scattering far exceeds in intensity the Rayleigh scattering due to $\mathrm{N}_{2}$ and $\mathrm{O}_{2}$ molecules in air. The sgcattered light intensity thus measures the dust concentration. Commercial dust monitors based on this principle are available [6]-[9], but those monitors which can accurately measure the concentration lower than $10 \mu \mathrm{g} / \mathrm{m}^{3}$ require frequent calibrations using dedicated equipment [7]. Such a high-sensitivity dust monitor is quite expensive inevitably.

A low-cost dust monitor is developed intended for use in ordinary households, offices, industries, schools, or hospitals. The principle of the real-time dust measurements is based on the conventional optical scattering method, but the optical system and signal processing are elaborated to realize the high-sensitivity while reducing the cost. In the following, the optical and electronic techniques and performances of a prototype monitor will be described.

\section{CONFIGURATION}

Fig. 1 shows a block diagram of the dust monitor developed. It consists of two main blocks; the optical system in which the laser beam interacts with dust to produce the scattered light and the signal processing unit for high-sensitivity detection of the scattered light. Each block will be next described in detail.

\section{A. Optical System}

As shown in Fig. 1 schematically, the optical system consists of a laser diode (LD), collimation lenses, pinhole slits, an integration room in a cylindrical shape, and a photo-detector (PD). The LD with wavelength of $810 \mathrm{~nm}$ and output power of $20 \mathrm{~mW}$ is used. After being collimated by lenses and slits, the laser beam enters into the integration room whose inner wall is coated with silver. The air whose dust concentration is to be 


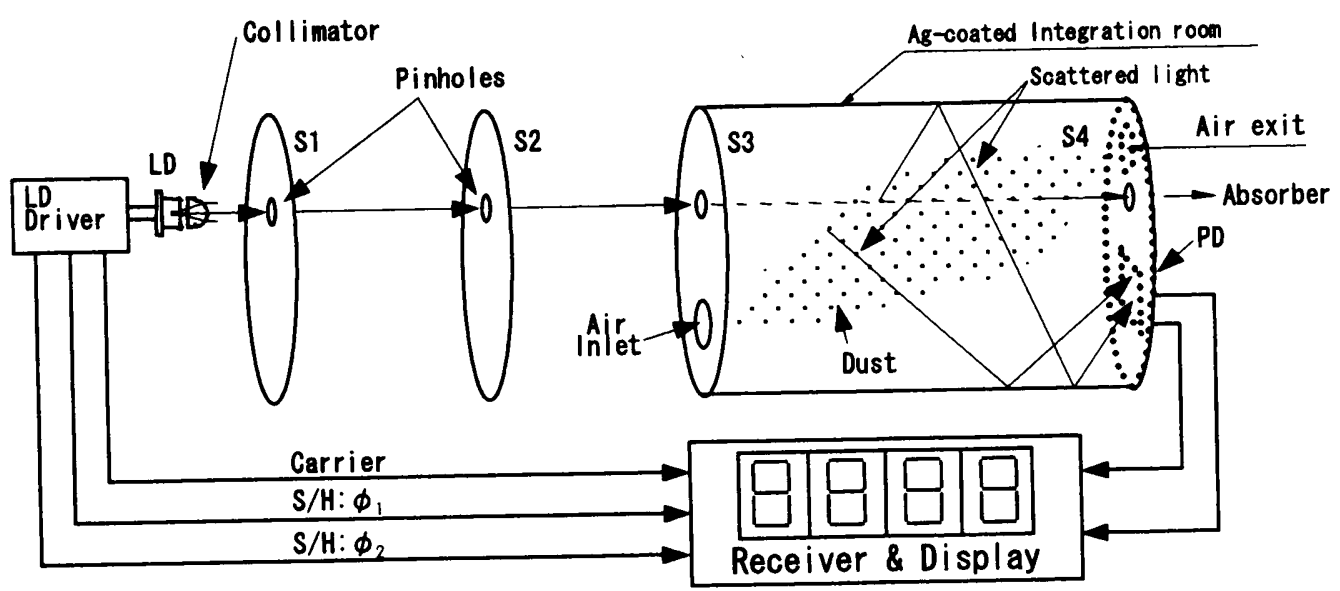

Fig. 1. Block diagram of the particle monitor.

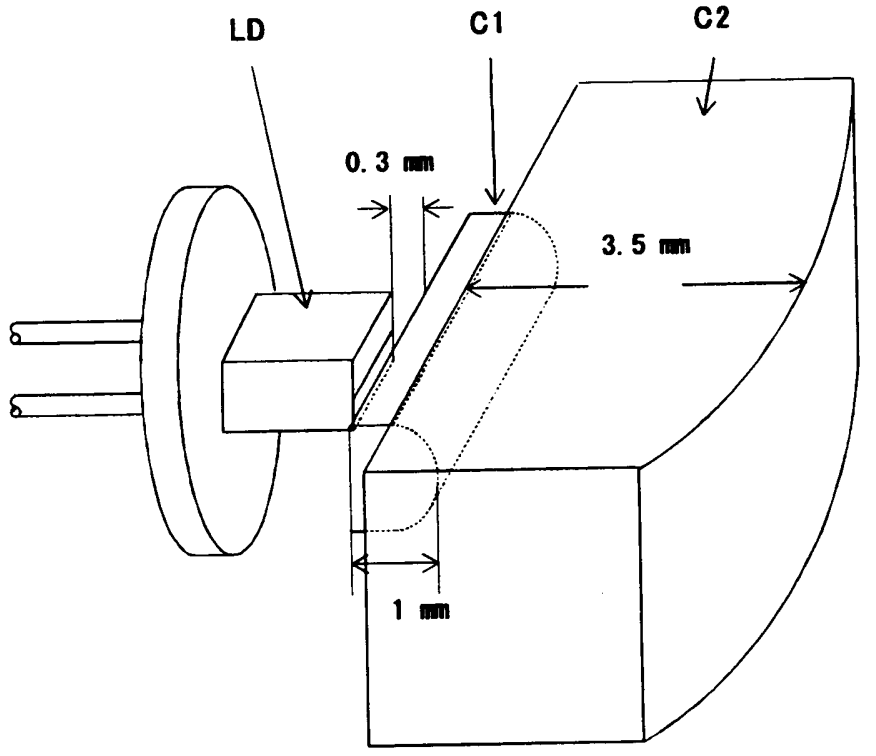

Fig. 2. Expanded view of the optical source.

measured is introduced into the integration room by suction. A small fan is used for suction. The laser beam travels $100 \mathrm{~mm}$ in the integration room while interacting with dust. This process produces scattering light which repeats multiple reflections by the Ag-coated inner wall until it eventually impinges on the PD mounted on the end plate of the integration room. The pin PD with the large aperture size $10 \times 10 \mathrm{~mm}$ is used for the high-efficiency detection. Because of the long interaction space and the high-efficiency integration of the scattered light, much higher sensitivity than that of a conventional monitor can be expected from the present optical system [5], [6].

Not only the scattered light but also the stray light due to diffraction and reflection is incident upon the PD. The ratio of scattered to stray light intensities then measures the signal-tonoise ratio (SNR), and suppression of the stray light is crucial for high SNR. One of the main sources of stray light is the surface irregularity of the collimation lens which acts as a secondary point source. The number of such point sources increases with the illuminated area. The most effective countermeasure is thus to place the collimation lens in close proximity to the LD.

Fig. 2 shows such a lens system. Because the radiation pattern from the LD is anisotropic, two cylindrical lenses with non- circular cross section are used to suppress aberration. Distance between the emitting surface of the LD and the flat back surface of the first cylindrical lens (C1) is $0.3 \mathrm{~mm}$. Center thickness of two cylindrical lenses $\mathrm{C} 1$ and $\mathrm{C} 2$ along the optic axis is $1 \mathrm{~mm}$ and $3.5 \mathrm{~mm}$, respectively. Refractive index of the lens material is 1.52 . $\mathrm{C} 1$ and $\mathrm{C} 2$ contact with each other.

A slit is also a source of stray light. If a collimated light beam impinges upon the edge of a pinhole, it emits a spherical wave, which spreads in all directions. To suppress such stray light, three slits are used. The collimated beam enters to the integration room after passing through two pinhole slits. Distance between $\mathrm{S} 1$ and $\mathrm{S} 2$, and $\mathrm{S} 2$ and $\mathrm{S} 3$ is $50 \mathrm{~mm}$. The collimated laser beam is limited to $1 \mathrm{~mm}$ in diameter by $\mathrm{S} 1$. S2 and $\mathrm{S} 3$ are larger in diameter than $\mathrm{S} 1$, thus reducing the diffraction due to $\mathrm{S} 3$ to a minimum.

Reflection from the absorber is another potential source of stray light. To prevent the reflected light from returning back to the integration room, the laser beam is pin-point focused to the pinhole S4.

\section{B. Signal Processing Units}

Potential interference sources which degrade SNR are sunlight, room light, and radio waves. To eliminate these influences, the modulation and the synchronous demodulation techniques are used. The modulation frequency of $125 \mathrm{kHz}$, referred to hereafter as the carrier frequency to avoid the confusion, is chosen for low-noise analog signal processing using op-amps. Another technique to achieve the high SNR is the averaging. Averaging over $N$ samples improves SNR by $10 \log N \mathrm{~dB}$. To provide the signal processing unit with the average function, the $L D$ is double-modulated. The intended SNR improvement is $30 \mathrm{~dB}$. The number $N$ of samples is thus 1000 . A sampling rate of 1000 samples per second (sps) is assumed. Such a slow rate is allowable because the regulations specify the dust concentration in terms of the average over $24 \mathrm{~h}$.

The LD driver and the PD circuit are designed based on the above-mentioned strategy. Fig. 3 shows the timing diagram of relevant signals. For easy implementation, the modulation signal is generated by dividing the $125 \mathrm{kHz}$ carrier using a binary counter. The period of the modulation signal is $1024 \mu \mathrm{s}$. To reduce the power dissipation in the $L D$, the duty ratio is set 


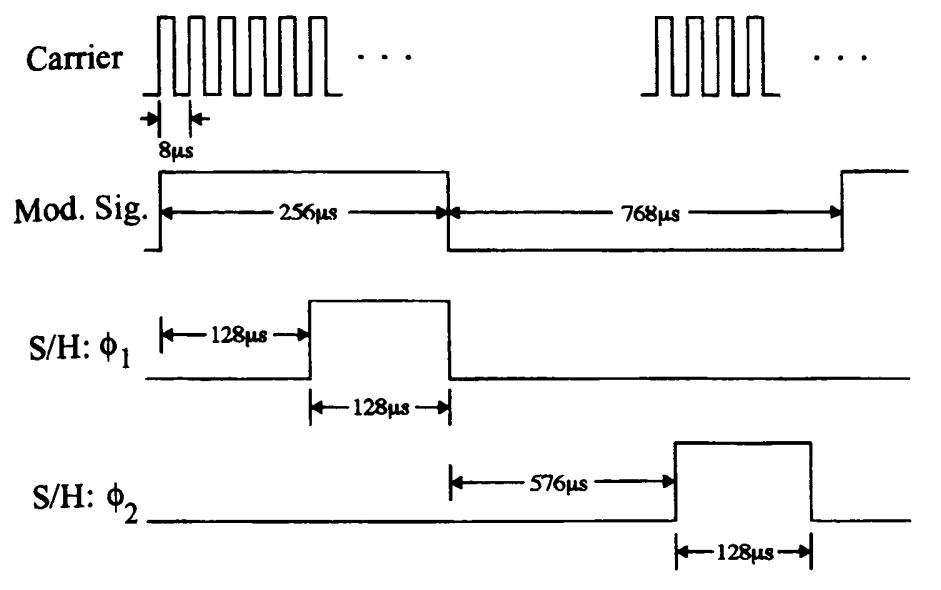

Fig. 3. Timing diagram of the modulation and demodulation signals.

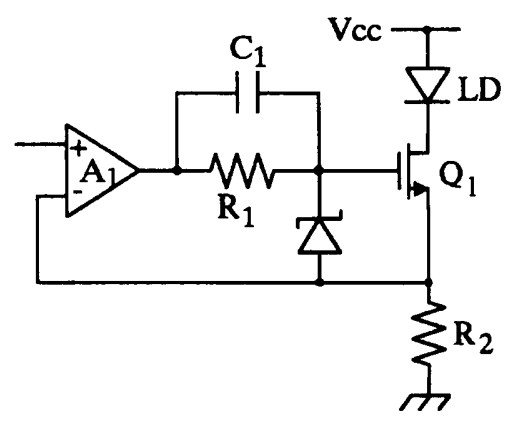

Fig. 4. Circuit diagram of the LD driving stage.

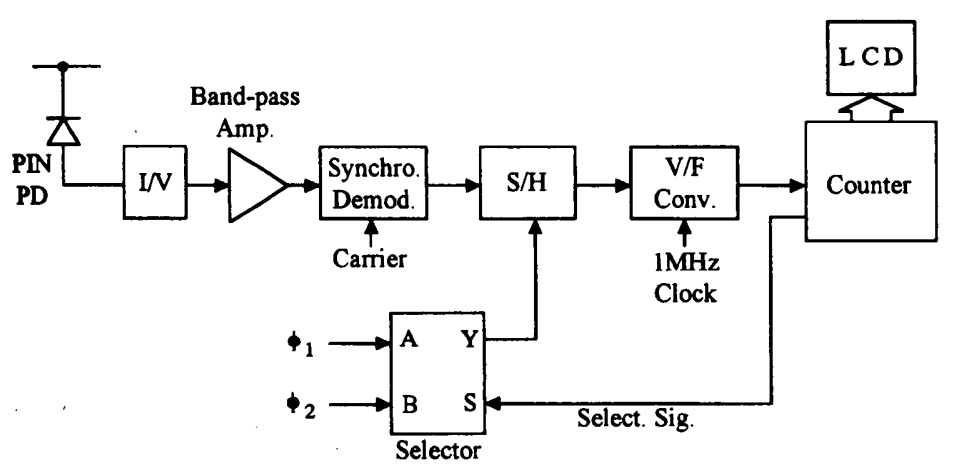

Fig. 5. Block diagram of the receiver.

at $25 \%$ and the double-modulation signal is produced by gating the carrier for $256 \mu \mathrm{s}$. The double-modulation signal thus produced is applied to the LD driving stage shown in Fig. 4. Consisting of the voltage-to-current converter with sink current capability up to $1 \mathrm{~A}$, this stage drives the LD by the constant current to stabilize the optical power. The driver also generates two sample/hold $(S / H)$ signals, one, $\phi_{1}$, for averaging the photo-detected signal and the other, $\phi_{2}$, for detecting the offset and the floor noise of the receiver, and send them to the receiver along with the carrier for synchronous demodulation.

Fig. 5 shows a block diagram of the receiver. For easy assembly, it is divided into three blocks: the front-end consisting of the current-to-voltage $(I / V)$ converter and the bandpass amplifier; the analog-to-digital conversion block consisting of the synchronous demodulator, the sample/hold $(S / H)$ circuit, and a voltage-to-frequency $(V / F)$ converter; and the counter block. The front-end converts the PD current induced by the scattered light into the voltage form and amplifies it. The pin PD with the

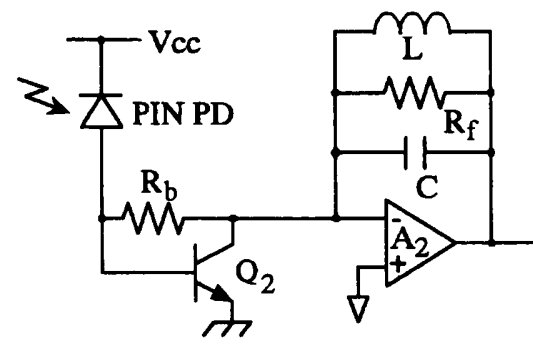

Fig. 6. Circuit diagram of the front-end.

large aperture $10 \times 10 \mathrm{~mm}^{2}$ used for high-efficiency detection of the scattered light is accompanied with the large capacitance of the order of $50 \mathrm{pF}$. The LD is modulated by a $125 \mathrm{kHz}$ carrier. Thus, the pin PD should be terminated by the low-impedance load. On the other hand, the high-sensitivity $I / V$ conversion requires the PD current to flow through a high-impedance termination. To meet these requirements, an elaborate $I / V$ converter shown in Fig. 6 is developed. The pin PD is terminated by the forward-biased base-emitter junction of a low-noise transistor $Q_{2}$. The PD current amplified by the current gain $\beta$ of $Q_{2}$ flows through the tank circuit tuned to $125 \mathrm{kHz}$, to be converted into the voltage. The conversion gain is thus $\beta R_{f}$. This $I / V$ converter is very stable because the inverting input terminal of op-amp $A_{2}$ is driven by the high-impedance current source and the conversion process is insensitive to the ambient light.

In the analog-to-digital conversion block, the output of the front-end is first multiplied by the $125 \mathrm{kHz}$ carrier and filtered by the second-order active low-pass filter. This process of synchronous demodulation improves SNR by $3 \mathrm{~dB}$ because the signal is in phase with the carrier while the noise generated in the front-end distributes uniformly. The demodulated signal is then applied to the $S / H$ circuit, to be converted into the digital equivalent. A low-cost $V / F$ converter is used for the digital conversion, though an analog-to-digital converter is a promising alternative. Two $S / H$ signals, $\phi_{1}$ and $\phi_{2}$, are sent from the LD driver. First, 1000 samples are sampled at $\phi_{1}$ timing, and the resultant frequency output of the $V / F$ converter is accumulated and averaged by the counter. The signal component in each sample is correlated but the noise component is uncorrelated. Thus, the average over 1000 samples improves SNR by $30 \mathrm{~dB}$. Next, $\phi_{2}$ is selected and the same operation is repeated by the counter to detect the offset and the noise of the receiver. The difference between these two counts is displayed on the LCD as the digital equivalent of the dust concentration.

\section{PROTOTYPE MONITOR}

Fig. 7 shows a picture of the optical system with the top plate removed and the signal processing units assembled on the printed-circuit boards. The counter block is housed in the metal case. An experimental setup for the performance evaluation is shown in Fig. 8. Seen to the right is a dust generator. The dust used is an internationally traceable standard latex $0.5 \mu \mathrm{m}$ in diameter. The fine latex particles are generated by suspension, atomizing, and drying processes, and then mixed with the clean air. The dust concentration is controlled by means of the air-flow rate. The dusty air is injected into the plastic box seen to the left through a hose, to replace the clean air in the box. The volume 


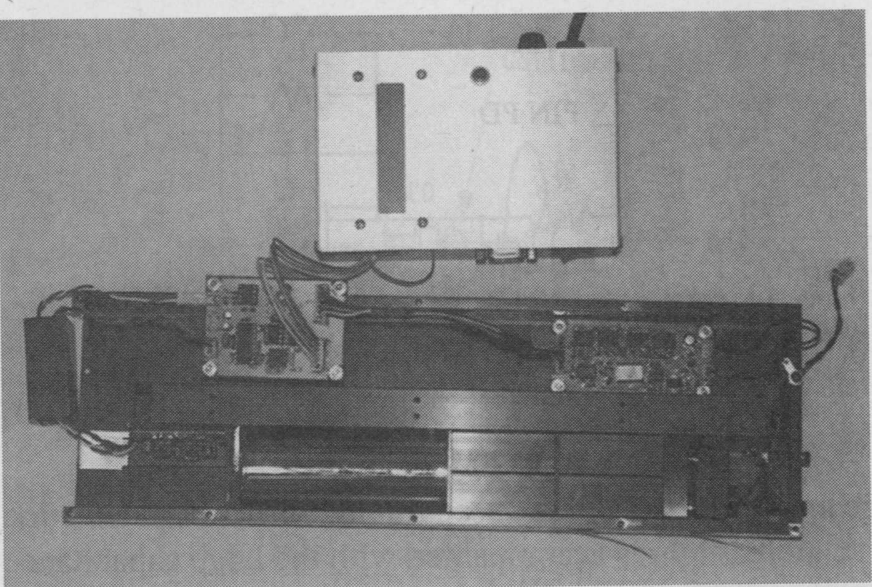

Fig. 7. Prototype particle monitor.

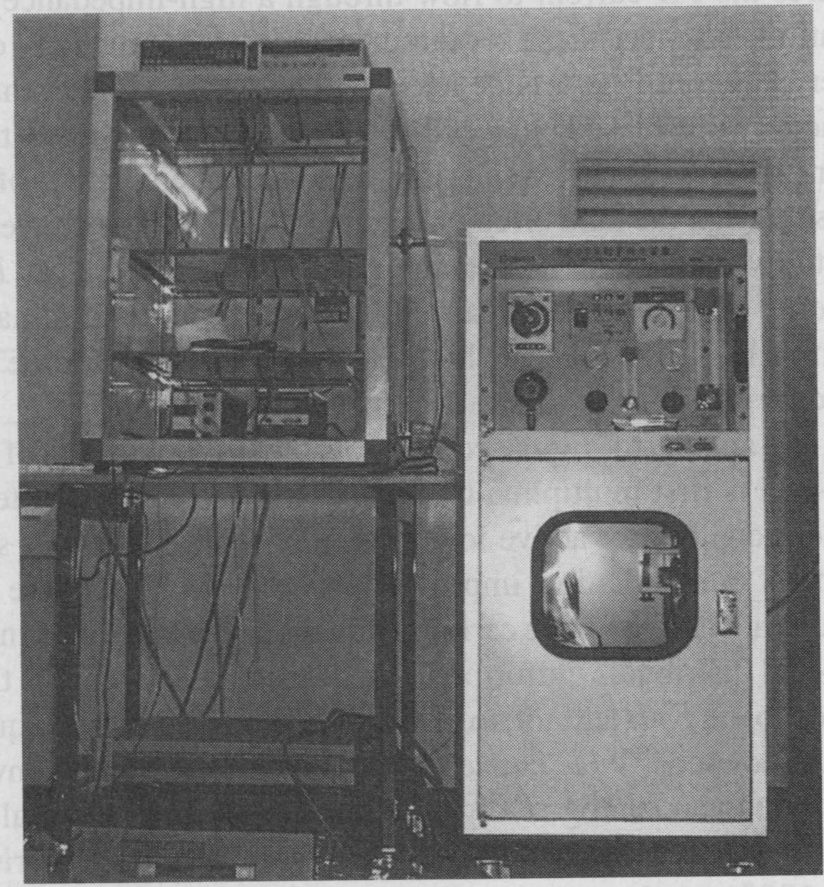

Fig. 8. Experimental setup for evaluating the performances of a prototype monitor.

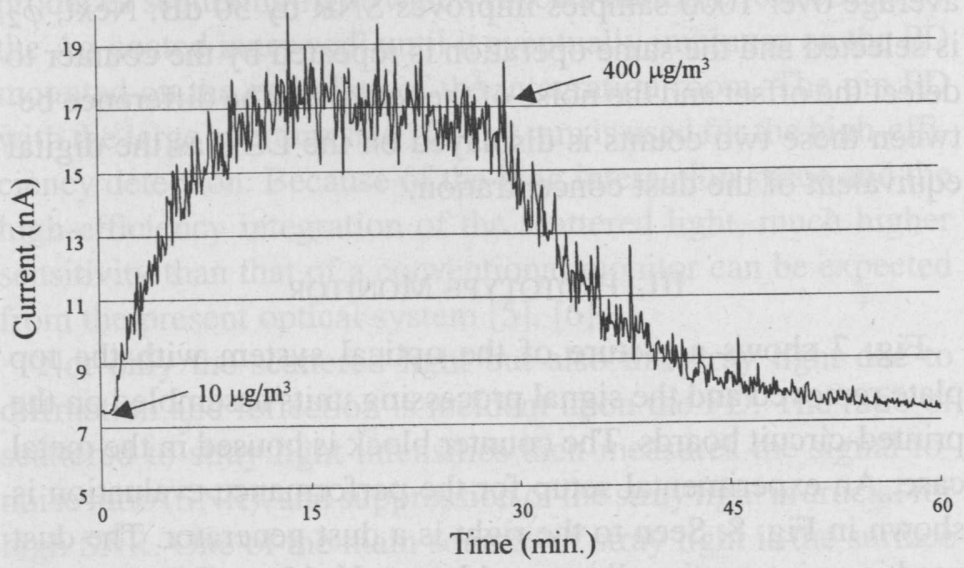

Fig. 9. PD current and the dust concentration in the course of time.

of the box is about 1801 and the flow rate of the dusty air is 30 $1 / \mathrm{min}$. The dust concentration in the box thus increases in the

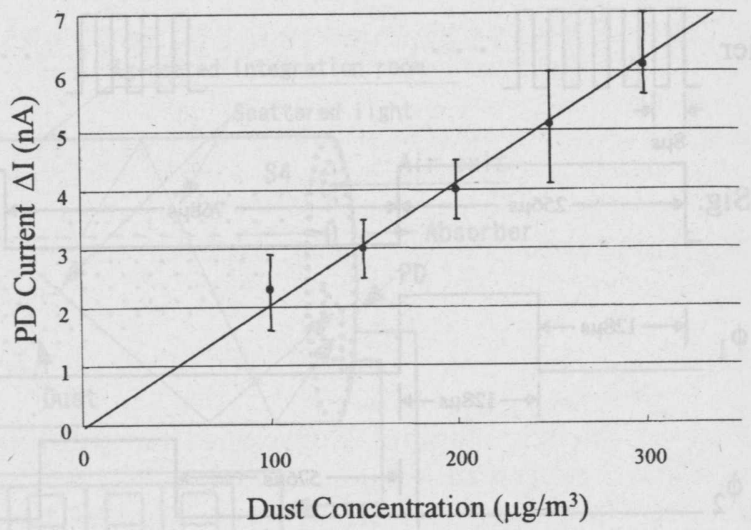

Fig. 10. Sensitivity of the optical system.

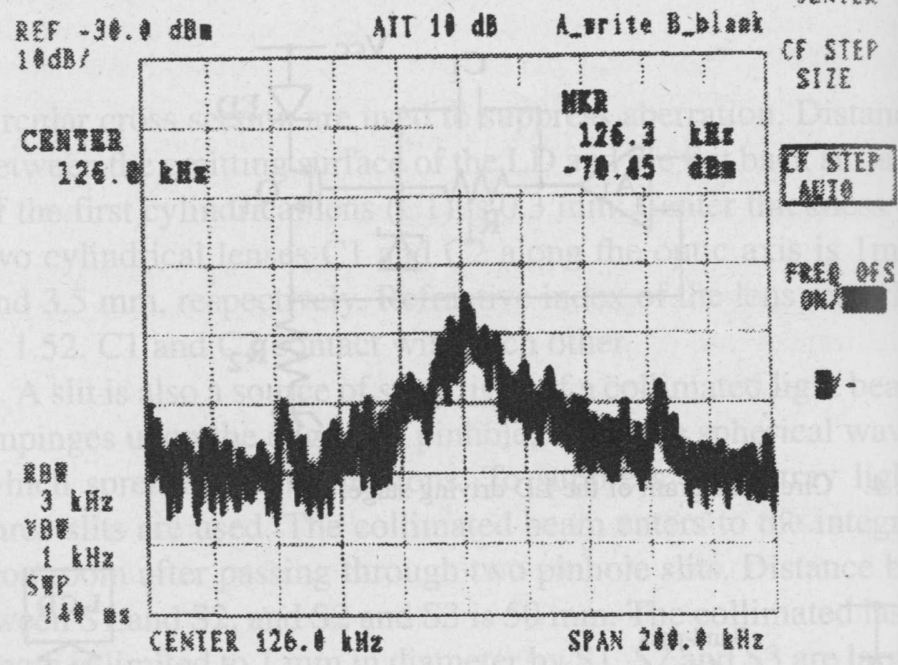

(a) Noise Floor

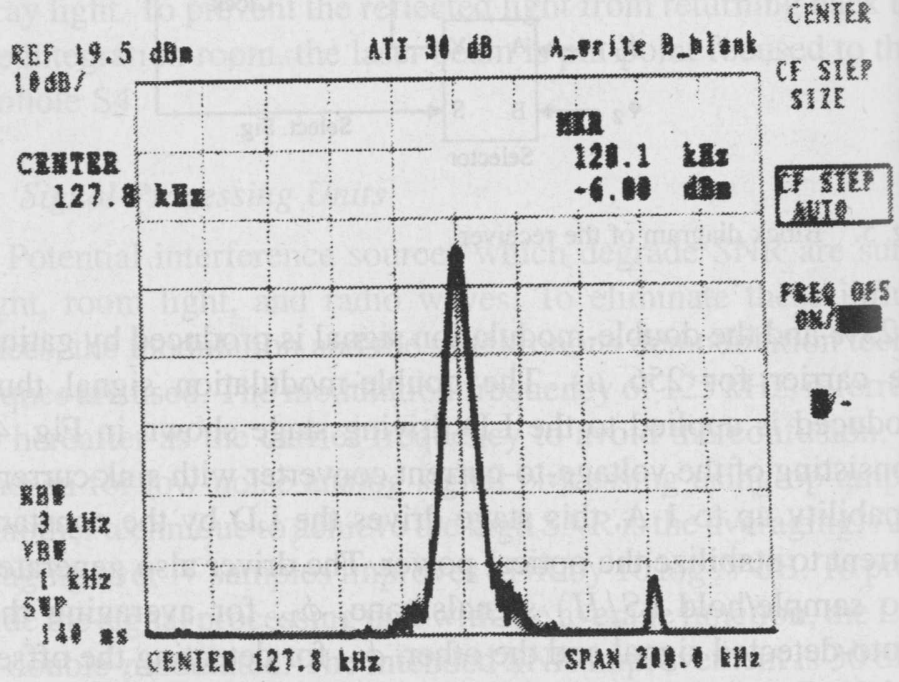

(b) Input signal current $=10 \mathrm{nA}$

Fig. 11. Output spectrums of the front-end.

course of time until it reaches that of the dusty air. A calibrated commercial monitor is used for measuring the dust concentration in the box. The minimum detectable dust concentration is $10 \mu \mathrm{g} / \mathrm{m}^{3}$. A prototype monitor is placed in the box along with the commercial monitor. 


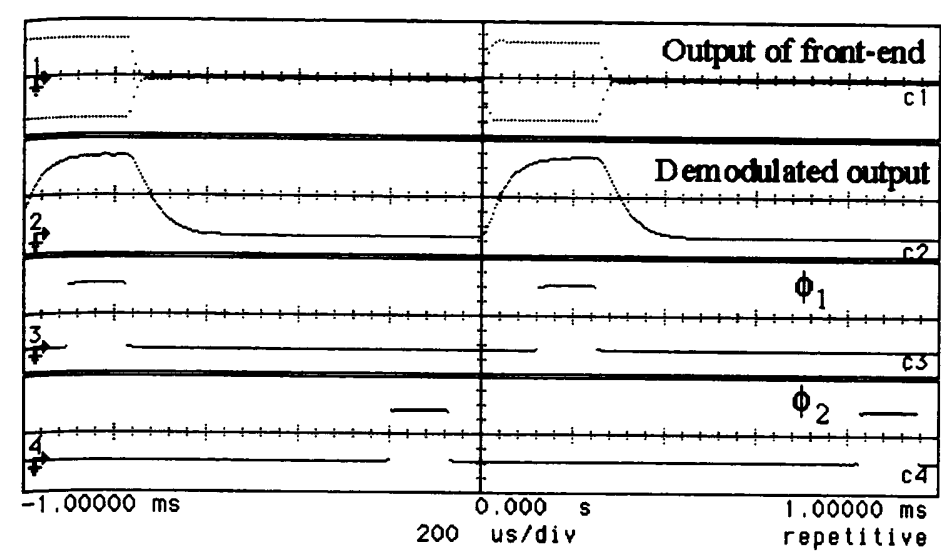

Fig. 12. Waveforms observed in a prototype monitor.

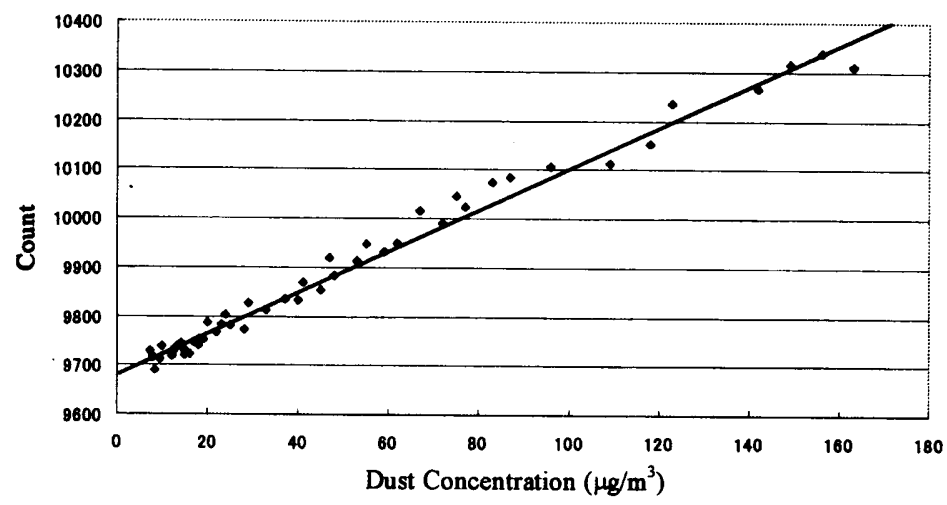

Fig. 13. Count as a function of dust concentration.

First, performances of the optical and signal processing units are evaluated individually. Fig. 9 shows the PD current and the monitored dust concentration in the course of time. In this measurement, the LD is not modulated but is driven by the dc current $80 \mathrm{~mA}$. The injection of the dusty air lasted for $30 \mathrm{~min}$ starting at $t=0$. Starting with the dark current $7.5 \mathrm{nA}$ at $t=0$, the PD current increases exponentially with time in accordance with the dust concentration in the box, to reach the steady-state value $17 \mu \mathrm{A}$ after around $12 \mathrm{~min}$, though a large instantaneous fluctuation is seen due to the nonuniform dust concentration. The average dust concentration monitored in the steady-state is $400 \mu \mathrm{g} / \mathrm{m}^{3}$. Fig. 10 shows the incremental change in the PD current against the dust concentration. The slope of the best-fitted straight line indicates that the PD current sensitivity is $20 \mathrm{pA} /\left(\mu \mathrm{g} / \mathrm{m}^{3}\right)$ approximately. The peak LD current when double-modulated is $200 \mathrm{~mA}$. The sensitivity of the optical system then increases to $50 \mathrm{pA} /\left(\mu \mathrm{g} / \mathrm{m}^{3}\right)$. Fig. 11 shows the SNR of the front-end. The noise floor is $-70 \mathrm{dBm}$ and the output level when driven by the sinusoidal signal with $10 \mathrm{nA}$ magnitude is $-6 \mathrm{dBm}$. The noise equivalent input signal current is thus $10 \mathrm{pA}$. From these individually evaluated performances, the minimum detectable dust concentration can be estimated to be $0.2 \mu \mathrm{g} / \mathrm{m}^{3}$. If the SNR improvement by averaging becomes effective, a dust concentration of as low as $7 \times 10^{-3} \mu \mathrm{g} / \mathrm{m}^{3}$ could be detected.
Overall performances of the prototype monitor were next evaluated by driving the LD by the double-modulation signal. Fig. 12 shows the output signal of the front-end (top trace), the synchronously demodulated signal (upper middle trace), and the two $S / H$ signals (lower middle and bottom traces). These observed waveforms and timing confirm that the monitor operates properly as designed. Fig. 13 shows the count displayed on the LCD as a function of dust concentration. Large deviations from the best-fitted straight line can be seen. This is due to the large instantaneous fluctuation in dust concentration, as can be seen in Fig. 9, which invalidates the SNR improvement by averaging. The lowest identifiable dust concentration is limited to $10 \mu \mathrm{g} / \mathrm{m}^{3}$ by the capability of the reference monitor. The slope of the best-fitted line in Fig. 13 indicates that the sensitivity is $4.0 /\left(\mu \mathrm{g} / \mathrm{m}^{3}\right)$. The fluctuation of the count due to noise is 20 . Therefore, the minimum detectable dust concentration of the prototype monitor can be evaluated to be $5 \mu \mathrm{g} / \mathrm{m}^{3}$.

\section{CONCLUSION}

A particle monitor developed for real-time measurements of dust concentration in living and working environments is described, and its performances are assessed. The performances of the optical system and signal processing unit evaluated individually have shown that a dust concentration lower than $0.2 \mu \mathrm{g} / \mathrm{m}^{3}$ is detectable. However, the minimum detectable dust concentration of the prototype monitor is $5 \mu \mathrm{g} / \mathrm{m}^{3}$. The deterioration is attributed to mainly misalignment of the optical system and the electromagnetic interference from the LD driver at the receiver front-end. An easily alignable optical source, shielding the signal processing units, miniaturization, replacing the $V / F$ converter by an $A / D$ converter for versatile functions, and costreductions constitute future work for the development of a commercial dust monitor.

\section{REFERENCES}

[1] M. O. Andreae, "Raising dust in the greenhouse," Nature, vol. 380, no. 6573, pp. 389-390, 1996.

[2] C. Keller, "Kontinuerliche Staubgehaltsmassung hinter Rauchgasreinigungen: Ein Ueberblick," Umwelt, vol. 23, no. 3, pp. 54-60, 1993.

[3] S. Chen and K. Watanabe, "A thermal environment monitor," in Proc. Int. Symp. Test and Measurement, June 1997, pp. 358-361.

[4] O. H. Peterson, T. Schneider, A. Abildgaard, N. P. Kloch, K. S Kongstad, and T. Bunch-Nielsen, "Measurement of particle contamination on indoor surface," J. Aerosol. Sci., vol. 23, pp. 555-558, 1992. Suppl. 1 .

[5] M. Abe, "A new dust monitor using paper filters," Working Environ., pp. $43-46,1993$. Special issue 25 , in Japanese.

[6] T. Kodachi, "An automatic dust concentration monitor based on nearinfrared scattering," Sens. Technol., vol. 12, pp. 204-207, June 1992. in Japanese.

[7] I. Enomoto, T. Otake, Y. Miyata, H. Mochizuki, T. Konishi, and K. Honma, "A new laser dust monitor," Working Environ., pp. 60-64, 1995. Special issue 29, in Japanese.

[8] E. Rudolf, "Einsatz eines direktanzeigenden Staubmessverfahrens bei der Ueberwachung von Giessereiarbeitsplaetzen," Giesserei, vol. 82, no. 22, pp. 822-824, 1995.

[9] M. Stromberg and H. Pucht, "Representative measurement of the smallest dust concentrations in exhaust gas channels," World Chem., vol. 27 , no. 10 , pp. 66, 69-71, 1996. 


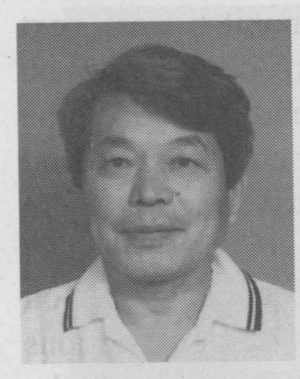

Kenzo Watanabe (M'74-SM'86-F'93) received the B.E. and M.E. degrees in engineering from Shizuoka University, Hamamatsu, Japan, in 1962 and 1966, respectively, and the Dr.Eng. degree from Kyoto University, Japan, in 1976.

He stayed at the University of California, Los Angeles, as a Visiting Professor from 1982 to 1983 . He is currently a Professor of the Research Institute of Electronics, Shizuoka University.

Dr. Watanabe received the Andrew R. Chi Best Paper Award and the IEEE Instrumentation and Measurement Society Award in 1984 and 1999, respectively, and the Saito Award from the Chion Institute in 1990 and the Takayanagi Memorial Award in 1994. He has also been designated an Emeritus Professor of Xidian University, Xi'an, China, in 1997. He is an Associate Editor of IEEE TRANSACTIONS ON INSTRUMENTATION AND MEASUREMENT.

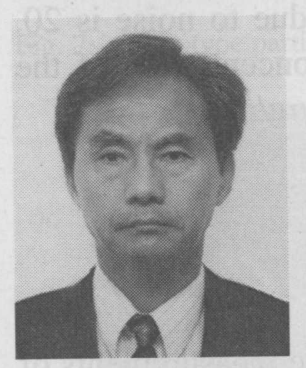

Tomuo Yamaguchi received the B.E. degree from Science University of Tokyo, Japan, in 1965 and the Dr. E. degree from University of Tokyo in 1973.

He was a Research Associate of the Faculty of Engineering, University of Tokyo, from 1965 to 1973. He moved to Shizuoka University, Hamamatsu, Japan, in 1974, where he is a Professor at the Research Institute of Electronics and engaged in optical measurements.

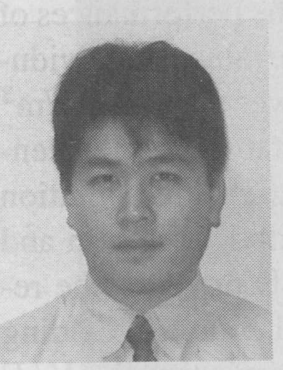

Masakatsu Nakayama graduated from Kanazawa Technical Senior High School, Kanazawa, Japan, 1990.

He joined the Research Institute of Electronics, Shizuoka University, Hamamatsu, Japan, as a Member of Technical Staff. He is engaged in hardware and software design and implementation of instrumentation and measurement systems.

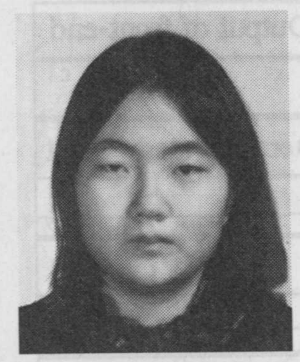

Yi-Zhu Gao received the B.E. degree from Beijing Information Technology Institute, Beijing, China, in 1997 and the M.E. degree from Shizuoka University, Hamamatsu, Japan, in 2000. She is currently pursuing the Dr. E. degree at Shizuoka University. Her topics of interest are III-V compounds and InAsSb for far infrared devices.
Tadashi Nagasawa received the B.E. and M.E. degrees in engineering from Shizuoka University, Hamamatsu, Japan, in 1986 and 1988, respectively.

$\mathrm{He}$ then joined Shizuoka Industrial Research Institute of Shizuoka Prefecture, where he is now engaged in the development of electronics technologies for industrial applications.

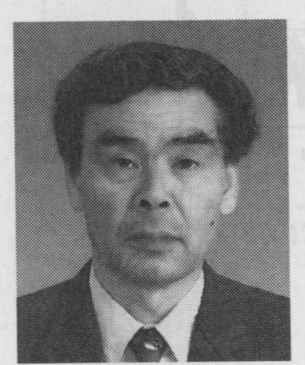

Taiyo Ozawa received the B.E. and M.E. degrees in engineering from Niigata University, Niigata, Japan, in 1968 and 1970 , respectively.

He then joined Turuoka College of Technology, Turuoka, Yamagata. In 1973, he moved to Shizuoka Industrial Research Institute of Shizuoka Prefecture, where he is now managing the development of electronics technologies for industrial applications. 